\title{
A PSICOPATOLOGIZAÇÃO DA INFÂNCIA NO CONTEMPORÂNEO: UM ESTUDO SOBRE A EXPANSÃO DO DIAGNÓSTICO DE "TRANSTORNO DE DÉFICIT DE ATENÇÃO E HIPERATIVIDADE"
}

CONTEMPORARY CHILDHOOD PSYCHO-PATHOLOGIZATION: A STUDY ON THE EXPANSION OF THE DIAGNOSES OF "ATTENTION DEFICIT DISORDER AND HYPERACTIVITY"

\section{LA PSICOPATOLOGIZACIÓN DE LA INFANCIA EN LA CONTEMPORANEIDAD: UN ESTUDIO SOBRE LA EXPANSIÓN DEL DIAGNÓSTICO DEL "TRASTORNO POR DÉFICIT DE ATENCIÓN E HIPERACTIVIDAD"}

\author{
Daniele de Andrade Ferrazza ${ }^{1}$ \\ Luiz Carlos da Rocha ${ }^{2}$
}

\section{RESUMO:}

A expansão da rotulação psiquiátrica não atinge apenas a população adulta, mas também as problemáticas relacionadas à infância têm sido capturadas pelos discursos e práticas do saber médico-psiquiátrico e transformadas em psicopatologias que tendem a ser tratadas com o principal recurso disponibilizado pela psiquiatria na contemporaneidade: os psicofármacos. este trabalho apresenta uma reflexão crítica sobre a expansão do diagnóstico de "transtorno de déficit de atenção e hiperatividade" (TDAH) em crianças e adolescentes e sobre a condução de terapêuticas medicamentosas que a tem acompanhado. concluí-se que os processos de psicopatologização da infância e de banalização da prescrição de psicofármacos estão relacionados à supervalorização da concepção biológica do sofrimento psíquico e a interesses econômicos dos grandes laboratórios farmacêuticos que por meio de diversas estratégias influenciam as práticas médicas, fatores que levam a exposição desses pacientes a possíveis efeitos colaterais e a riscos de estigmatização que devem ser considerados.

Palavras-chave: Psicopatologização. Infância. Medicalização. Transtorno de déficit de atenção e hiperatividade (TDAH).

\footnotetext{
${ }^{1}$ Doutoranda em Psicologia pelo Programa de Pós-Graduação da Faculdade de Ciências e Letras da Universidade Estadual Paulista Júlio de Mesquita Filho (UNESP), Assis-SP.

E-mail: danieleferrazza@yahoo.com.br

${ }^{2}$ Doutor em Psicologia pela Universidade de São Paulo (USP). Professor do curso de Psicologia da Faculdade de Ciências e Letras da Universidade Estadual Paulista Júlio de Mesquita Filho (UNESP), Assis-SP. E-mail: Icrocha@assis.unesp.br
} 


\section{ABSTRACT:}

The expansion of psychiatric labeling does not reach only the adult population, but also the problematic related to childhood have been captured by the speeches and practices of the medical-psychiatric knowledge and turned into psychopathologies which tend to be treated with the main resource made available by psychiatrics in the present times: the psychotropic drugs. This work presents a critical thinking on the expansion of the diagnoses of "attention deficit disorder and hyperactivity" (ADHD) both in children and teenagers and on the conduction of drug therapies. It follows that the processes of childhood psycho-pathologization and the trivializing of psychotropic drug prescription are related to the overvaluation of the biological conception of psychic suffering and to the economic interests of the great pharmaceutical laboratories which by means of several strategies influence the medical practices, factors that lead to exposure of these patients to possible side effects and the risks of stigmatization that must be considered.

Keywords: psycho-pathologization; childhood; medicalization; "attention deficit disorder and hyperactivity" (ADHD).

\section{RESUMEN:}

La expansión del etiquetado psiquiátrico no afecta solamente a la población adulta, sino también las problemáticas relacionadas a la infancia han sido capturadas por los discursos y prácticas del saber médico-psiquiátrico y transformadas en psicopatologías que tienden a ser tratadas con el principal recurso disponible por la psiquiatría contemporánea: los psicofármacos. Este trabajo presenta una reflexión crítica sobre la expansión del diagnóstico de "Trastorno por Déficit de Atención e Hiperactividad" (TDAH) en niños y adolescentes, y sobre la conducción de terapéuticas medicamentosas que le han acompañado. Se concluye que los procesos de psicopatologización de la infancia y de banalización de la prescripción de psicofármacos están relacionados a la sobrevaloración de la concepción biológica del sufrimiento psíquico, y a los intereses económicos de los grandes laboratorios farmacéuticos que por medio de diversas estrategias influencian las prácticas médicas, factores que exponen estos pacientes a posibles efectos colaterales y a riesgos de estigmatización que deben ser considerados.

Palabras clave: Psicopatologización. Infancia. Medicalización. "Trastorno por Déficit de Atención e Hiperactividad" (TDAH).

\section{INTRODUÇÃO}

A expansão da rotulação psiquiátrica não atinge apenas a população adulta, mas também as problemáticas relacionadas à infância têm sido capturadas pelos discursos e práticas do saber médico-psiquiátrico e transformadas em psicopatologias que passam a ser tratadas com o principal recurso disponibilizado pela psiquiatria na contemporaneidade: os psicofármacos. Este trabalho aborda esse processo de psicopatologização da infância, que também tem atravessado os 
espaços escolares, e apresenta uma reflexão crítica sobre a expansão do diagnóstico de "Transtorno de Déficit de Atenção e Hiperatividade" (TDAH) e da condução de terapêuticas medicamentosas que Ihe tem sido correlativa.

A primeira parte deste artigo aborda a questão do reducionismo biológico do sofrimento psíquico, esteio da estereotipia psiquiátrica que tem encontrado nas variadas manifestações de mal-estar e em comportamentos virtualmente inadequados, sinais e sintomas de alguma psicopatologia. Aqui também serão pontuados os interesses que, nessa expansão da medicalização, consorciam aspectos corporativos da psiquiatria ao expansionismo da indústria farmacêutica. $A$ segunda parte procura explorar o tema das origens dessa atual psicopatologização da infância e problematiza a atual expansão do diagnóstico de TDAH, particularmente no âmbito das instituições educacionais e congêneres. Por fim, serão apresentadas algumas das implicações desse diagnóstico psiquiátrico na infância, essa fase considerada tão fundamental para o desenvolvimento humano pela psicologia.

\section{A PSICOPATOLOGIZAÇÃO DA VIDA COTIDIANA E A EXPANSÃO DAS PRÁTICAS MÉDICAS}

Atravessamos, atualmente, uma circunstância em que elementares sinais de sofrimento psíquico podem ser rotulados como uma patologia cujo tratamento costuma ser a administração de psicofármacos. Nessa prática, pode-se perceber uma tendência geral da medicina em transformar em questão médica aquilo que é da ordem do social, do econômico, do político, do cultural (AMARANTE, 2007). Conforme ponderou Birman (1978), acompanhando o fundamental exame histórico de Castel (1978), esse procedimento pode ser visto como manifestação atualizada de uma tendência perceptível desde a segunda metade do século XIX europeu, quando a extensão moreliana do tratamento moral alienista, do interior dos manicômios às concentrações urbanas da pobreza, assinalou a vocação de medicina de população da ainda nascente psiquiatria. Depois, ao longo dos tempos, esse restrito saber especializado, já sob marcante demanda corporativa, foi se apropriando de inúmeras manifestações da existência humana transformando-as em 
aspectos que seriam submetidos, sob variadas rotulações psicopatológicas, às práticas do organicismo médico.

Nesse processo de psicopatologização da vida cotidiana, a descoberta dos primeiros psicofármacos nos anos 50 , e sua proliferação a partir dos anos 80 do século passado, cumpriria o papel de, enfim, fornecer algum recurso realmente médico a uma concepção organicista sobre o caráter do sofrimento psíquico que acompanha a psiquiatria desde o fisicalismo do século XIX. Desde então, alavancada pela banalização da prescrição de psicofármacos, a psiquiatria pode ampliar seu alcance para além do tratamento exclusivo da loucura e apresentar-se como o domínio de saber (FOUCAULT, 2001; 1979) credenciado a diagnosticar e tratar, virtualmente, qualquer mal-estar cotidiano (FERRAZZA, et. al., 2010). Podese, então, reconhecer um verdadeiro processo de psicopatologização da vida social (AGUIAR, 2003; BIRMAN, 2000), fato correlato a uma tendência normativa alçada pela supervalorização de concepções biológicas do sofrimento psíquico fundadas em hipóteses neurológicas e genéticas, fatores que incentivam e generalizam o tratamento baseado em recursos farmacológicos. (LEGNANI; ALMEIDA, 2008; GUARIDO, 2007; QUINET, 2001; BOGOCHVOL, 2001). Nessa configuração, conferiu-se um oportuno efeito de verdade científica à concepção de que a única explicação válida para qualquer tipo de desconforto psíquico é a descrição fisicalista, sob a qual a vida psíquica do homem é reduzida a sua estrutura biológica (COLLARES; MOYSÉS, 1994).

Podemos observar que, ainda no século XIX, grande parte dos discursos da medicina psiquiátrica estava fundamentada na idéia de um determinismo biológico, onde comportamentos considerados inadequados eram caracterizados como um problema orgânico e hereditário (CAPONI, 2007). Esse tipo de concepção formulada em tempos passados viria a se fortalecer com o surgimento das tecnologias de mapeamento genético e da descoberta de drogas psicofarmacológicas que poderiam ajustar aqueles "comportamentos indesejados" sobre os quais a psiquiatria acalentava associações hipotéticas com disfunções cerebrais. Sobre as críticas a essa concepção, Guarido (2007) faz o seguinte comentário:

Na sua grande maioria, as análises focam a presença atual de diagnósticos psiquiátricos que estabelecem bases biológicas para os sofrimentos psíquicos, aproximando os fenômenos mentais das doenças orgânicas, ou seja, vê-se um abandono crescente da descrição causal e de sentido dos sintomas apresentados pelo sujeito em benefício de uma noção em que os 
sintomas são reconhecidos como manifestações de desordens da bioquímica cerebral (GUARIDO, 2007, p. 153).

Nessa configuração, novos diagnósticos vêm sendo criados pela psiquiatria conforme as transformações das problemáticas do mundo contemporâneo. Síndromes do Pânico, Depressões, Toxicomanias e, no âmbito da infância, "Transtorno de Déficit de Atenção e Hiperatividade" são rotulações diagnósticas da atualidade a que cada vez mais pessoas são submetidas.

Longe de negar que muitas experiências e sensações possam produzir sofrimentos e mal-estares difíceis de serem enfrentados, chama atenção o fato de problemáticas comuns da vida cotidiana de uma expressiva maioria da população serem transformadas em diagnósticos psiquiátricos e, consequentemente, ficarem afeitos à medicação psicofarmacológica como única medida de enfrentamento e de entendimento. Essa parece ser uma tendência da prática médica atual, onde o malestar cotidiano "não é mais compreendido como uma experiência subjetiva de um determinado indivíduo, mas sim, como uma entidade nosológica que o acomete." (RODRIGUES, 2003, p. 15).

Nesse processo de psicopatologização do homem desenvolve-se uma transformação do sofrimento psíquico em uma patologia cujo tratamento seria a continuada administração de medicamentos. Para além de um reducionismo ofensivo à complexidade da existência humana, Legnani e Almeida (2008, p. 3) sugerem que "a atuação medicamentosa da prática médica vem sendo convocada para atenuar e apaziguar os conflitos, sejam estes de caráter social, político, ou mesmo psíquicos."

\section{SOBRE AS RELAÇÕES DA PSIQUIATRIA COM A INDÚSTRIA FARMACÊUTICA}

$\mathrm{Na}$ segunda metade do século XX, com o desenvolvimento da psicofarmacologia e a da neurologia, a psiquiatria logrou legitimar-se como ciência biológica, sonho perseguido desde o século XIX (BIRMAN, 2000). A tendência à banalização da prescrição de psicofármacos, entretanto, não pode ser vista como um processo isento de interesses corporativos nem sempre atentos ao bom cuidado clínico e às delicadas condições do sujeito em sofrimento psíquico. Demandas 
empresariais de expansão e lucro da indústria farmacêutica são fatores que devem ser considerados na avaliação do processo de medicalização da existência humana, como pondera Guarido (2007):

A produção de saber sobre o sofrimento psíquico encontra-se associada à produção da indústria farmacêutica de remédios que prometem aliviar os sofrimentos existenciais. $\mathrm{O}$ consumo em larga escala dos medicamentos e $\mathrm{O}$ crescimento exponencial da indústria farmacêutica tornam-se elementos indissociáveis do exercício do poder médico apoiado em um saber consolidado ao longo do século XX. (GUARIDO, 2007, p.158).

A partir da década de 50, quando foi sintetizada a clorpromazina, o primeiro psicofármaco utilizado no tratamento da esquizofrenia, as indústrias de medicamentos passaram a investir fortemente na produção de drogas para o tratamento psiquiátrico (GUARIDO; VOLTOLINI, 2009). O resultado, marcadamente mercadológico, foi o surgimento de um número excedente de psicofármacos no mercado. Conforme Angel (2007), essa grande quantidade de medicamentos raramente é constituída de produtos realmente inovadores, ainda que sempre sejam assim anunciados. Muitas vezes, são versões, apresentadas sob novas roupagens, de medicamentos já existentes no mercado. Ainda que apresentados como inovações farmacológicas, conforme Cordeiro (1985), nem sempre cumprem funções diferentes ou melhores do que aqueles que já estavam sendo comercializados, salvo pelas nomenclaturas atrativas e pelos altos preços que lhes conferem a aparência de produtos de ponta.

Conforme Angell (2007), os laboratórios farmacêuticos freqüentemente anunciam novas doenças para se encaixarem nos medicamentos que pretendem lançar. Pode-se, dessa forma, acompanhando as noções de Foucault $(2001 ; 1979)$ sobre a produção de efeitos de verdade por práticas sociais historicamente constituídas, compreender a proliferação de diagnósticos psiquiátricos como construções operadas por uma medicina que privilegia a consolidação de seu poder e por uma indústria farmacológica que investe em seus próprios interesses empresariais. 


\section{A PSICOPATOLOGIZAÇÃO DA INFÂNCIA}

A transformação do sofrimento psíquico em doença e seu enquadramento no âmbito da variedade de rotulações diagnósticas produzida pela psiquiatria apresenta sinais de estender-se, atualmente, a uma infância que ainda vinha sendo poupada dos veredictos psicopatológicos e da prescrição de psicofármacos que costuma acompanhá-los. As condutas da infância que são consideradas inadequadas e/ou indesejáveis estão sendo transformadas, pelo saber psiquiátrico, em manifestações sintomáticas de psicopatologias. Atualmente, a determinação diagnóstica que mais tem atingido crianças e adolescentes é o "Transtorno de Déficit de Atenção e Hiperatividade" (TDAH), para o qual a psiquiatria vem recomendando, principalmente, a administração de psicofármacos (BRZOZOWSKI; CAPONI, 2010; LEGNANI; ALMEIDA, 2008; GUARIDO, 2007). Como nem sempre a infância esteve sob o olhar da psiquiatria, é conveniente uma reflexão sobre sua entrada no âmbito desse domínio de saber.

$\mathrm{Na}$ compreensão do processo de constituição da psicopatologização da infância, Foucault (2006) nos mostra que a criança passa a ser objeto de intervenção psiquiátrica bem depois do adulto:

Poderíamos naturalmente esperar que essa psiquiatrização da infância tenha
se feito por dois caminhos que parecem como que ditados de antemão: de
um lado, o caminho que seria o da descoberta da criança louca; o outro
caminho seria o de fazer a infância emergir como lugar de fundação, lugar de
origem da doença mental. Ora, tenho a impressão de que não foi exatamente
assim que as coisas aconteceram. De fato, parece-me que a descoberta da
criança louca foi afinal uma coisa tardia e muito mais o efeito secundário da
psiquiatrização da criança do que seu lugar de origem. (FOUCAULT, 2006, p.
256).

Conforme Foucault (2006), até o fim do século XIX, a psiquiatria, quando realizava uma anamnese com o adulto considerado doente mental, não pretendia tratar sua loucura a partir dos acontecimentos da infância. Almejava apenas captar sinais que confirmassem a predisposição hereditária da doença. Dessa forma, a psiquiatrização da infância teria suas origens não exatamente na criança considerada louca, mas em sinais de um desenvolvimento hipoteticamente comprometido, como resultado das concepções da época sobre a hereditariedade da loucura. Nesse sentido, Foucault (2006, p. 257) considera que "foi por intermédio da 
criança não-louca que se fez a psiquiatrização da criança e, a partir daí, que se produziu essa generalização do poder psiquiátrico" que se estenderia à infância.

Seria, então, a partir do exercício de um jogo de suposições etiológicas que a psiquiatria iria se autorizar a intervir em qualquer mal-estar ou comportamento infantil considerado indesejado para disseminar seus discursos e práticas sobre uma pretensa infância problemática a ser atendida pelos seus recursos. Nessa perspectiva, aquelas crianças que não se adaptavam às regras e normas da sociedade vigente estariam, então, sujeitas aos discursos e práticas normatizadoras da medicina psiquiátrica que, conforme expõe Caponi (2007, p. 344), "possuem ainda hoje, como ocorreu no início do século $X X$, diagnósticos ambíguos e imprecisos, terapêuticas de eficácia duvidosa e efeitos colaterais imprevisíveis".

\section{A "BOLA DA VEZ" DO AVANÇO DA PATOLOGIZAÇÃO DA INFÂNCIA: 0 "TRANSTORNO DE DÉFICIT DE ATENÇÃO E HIPERATIVIDADE"}

Assim como aconteceu a partir dos anos 70 nos Estados Unidos da América, nosso país vem assistindo, hoje, a uma avalanche de diagnósticos de "Transtorno de Déficit de Atenção e Hiperatividade":

[...] uma das descrições médicas mais utilizadas para dar vazão ao mecanismo de psicopatologização das dificuldades que comparecem na infância, em uma concepção biologizante do desenvolvimento e do psiquismo humanos. As primeiras descrições médicas sobre o TDA/H aparecem no início do século XX, mas é a partir da década de 1970, principalmente no contexto norte-americano, que essa descrição passa a ganhar um grande destaque nos diagnósticos das crianças e adolescentes em processo de escolarização. (LEGNANI; ALMEIDA, 2008, p.5).

Eleita como a "bola da vez" da investida psicopatologizante sobre a infância, esse diagnóstico, atualmente, vem sendo objeto de divulgação alarmista na mídia, através de reportagens e sites especializados no assunto. É digno de nota o efeito potencializador dessa campanha na preocupação da opinião pública pouco informada, notadamente sobre aqueles que têm filhos em idade escolar e, como é comum, defrontam-se com os corriqueiros problemas de suas crianças em fase de adaptação educacional. Essas dificuldades tão comuns, em sua grande maioria, podem ser adequadamente atendidas por recursos pedagógicos perfeitamente ao alcance dos profissionais de educação. No entanto, algumas escolas têm distribuído manuais e cartilhas (MAIA; VEREJÃO, 2002; ROHDE; BENCZIK, 1999; CARTILHAS 
DA ABDA on-line, 1999) para pais e professores com explicações do que é o TDAH e de como identificar os sintomas de desatenção, inquietação e impulsividade nos possíveis "portadores do transtorno", que é apresentado como uma recente descoberta da psiquiatria infantil. Talvez seja por essa difusão marcada por interesses comerciais da indústria farmacológica que cada vez mais crianças que apresentam quaisquer dificuldades de aprendizagem e/ou "comportamentos inadequados" vêm sendo rotuladas com esse diagnóstico estigmatizante e de tratamento perigoso.

Assiste-se, na verdade, não a um aumento de incidência de uma doença, mas a um surto de diagnósticos de TDAH operado sobre crianças com problemas perfeitamente normais para a faixa de idade (GUARIDO; VOLTOLINI, 2009). Essa percepção costuma ser refutada por setores da psiquiatria vinculados aos fabricantes dos medicamentos para esse diagnóstico, que exercitam o parecer conveniente de que essa doença seria, conforme Gomes et. al. (2007), subestimada, e que agora sua pronta detecção pode conduzir a criança ao tratamento adequado. É interessante notar que, não sem motivos, o tratamento adequado é justamente aquele medicamento fabricado por seus patrocinadores.

\section{SOBRE O PRINCÍPIO ATIVO DESSE SURTO DE MEDICAÇÃO}

O principal tratamento determinado àqueles que são diagnosticados com TDAH é a prescrição de um medicamento psicoestimulante, o cloridrato de metilfenidato, que apresenta, entre outros, o nome comercial de "Ritalina" ou "Concerta". Apesar das diversas pesquisas publicadas na atualidade tentando alertar pais e professores sobre os prejuízos na vida daqueles que apresentam sintomas do TDAH (ROHDE et. al., 2000; GOMES et. al., 2007), há poucos trabalhos que advertem sobre os efeitos colaterais do uso do metilfenidato. Segundo Barros (2008), dentre os diversos efeitos adversos, destacam-se insônia, taquicardia, dores abdominais, anorexia, perda de peso. A propósito, Telles (2000) comenta:

Muitas das drogas mais usadas, como Prozac, Ritalina e Atensina, sequer foram testadas quanto a sua segurança ou eficácia quando tomadas por pacientes tão jovens. Essencialmente, os médicos que receitam esses medicamentos estão presumindo que eles funcionarão para quem mal

R. Inter. Interdisc. INTERthesis, Florianópolis, v.8, n.2, p. 237-251, Jul./Dez. 2011 
deixou as fraldas, assim como funcionam comprovadamente em adultos e crianças mais velhas. "Precisamos descobrir a validade do uso de medicação nessas crianças. E será que elas merecem o remédio que tomam?", questiona Julie Magno Zito, professora da Faculdade de Farmacologia da Universidade de Maryland. Ela é especialmente cética quanto ao uso de Ritalina para combater deficit de atenção em crianças pequenas: "o que é um nível de desatenção fora do normal para alguém de dois anos?" (TELLES, 2000, p. 5).

É também de relevante destaque, o fato de que aqueles manuais produzidos pelas associações médicas que visam divulgar o TDAH para pais e professores são, na grande maioria das vezes, patrocinados por laboratórios farmacêuticos que sintetizam o metilfenidato (MAIA; VEREJÃO, 2002; ROHDE; BENCZIK, 1999). Nesse quadro, é conveniente considerar que a influência dos laboratórios farmacêuticos nas práticas e nos discursos psiquiátricos sobre o TDAH possa estar influenciada pelos interesses econômicos das grandes corporações de fabricantes desse produto, cuja produção tem mostrado um crescimento impressionante: De acordo com o último relatório da Organização das Naçães Unidas (ONU)
sobre produção e consumo de psicotrópicos (ONU, 2008), em que os dados
sobre o metilfenidato são apresentados separadamente dos outros
estimulantes, sua produção mundial no ano de 2006 chegou a quase 38
toneladas. Destas 38 toneladas, 34,6 foram produzidas pelos EUA, que são
não somente os maiores fabricantes, mas também os maiores
consumidores. A maior parte do que os EUA produzem é para uso interno.
O consumo nos EUA vem crescendo a cada ano, e hoje representa 82,2\%
de todo metilfenidato consumido no mundo." (ORTEGA, et. al, 2010).

\section{SOBRE AS PROPALADAS "VANTAGENS" DA MEDICAÇÃO PRECOCE DAS CRIANÇAS}

Para os psiquiatras da Associação Brasileira de Déficit de Atenção (ABDA), existem diversas vantagens em medicar as crianças e adolescentes diagnosticados com esse transtorno, pois, "os sintomas do transtorno podem prejudicar o desenvolvimento emocional da criança e até levá-la ao consumo de substâncias químicas, como álcool e drogas." (ROHDE, et. al., 2000). Os argumentos de que esse medicamento poderia evitar que essas crianças e adolescentes se tornassem, mais tarde, usuários de drogas ilícitas não foram comprovados e costumam ser apresentados como meras especulações (LEGNANI; ALMEIDA, 2008). Aparece, também, a opinião de que ao se transformar as dificuldades em algo de natureza biológica se evitaria a implicação moral do sujeito e, conseqüentemente, o problema 
seria algo do âmbito e do controle médico, como nos aponta Legnani e Almeida (2008):

\begin{abstract}
A argumentação é a de que o efeito dos psicoestimulantes também regula o self, pois o adolescente experimentaria um alívio ao saber-se portador de uma desordem passível de tratamento. Desse modo, este saber elevaria a auto-estima do paciente por desfazer a conotação moral acerca do problema, geradora de culpa. (LEGNANI; ALMEIDA, 2008, p. 8).
\end{abstract}

Em resumo, parece que querem nos fazer crer que a drogadição psiquiátrica precoce da infância poderia prevenir uma aproximação posterior do adolescente às drogas e que deve fazer bem para as crianças sentirem-se doentes e em tratamento psiquiátrico. Chega a ser risível, se não fosse trágico.

\title{
CONSIDERAÇÕES FINAIS
}

A rotulação diagnóstica de TDAH e a expansão da prescrição de psicofármacos para as crianças estão afeitas a vários fatores, como a supervalorização da concepção biológica do sofrimento psíquico e a determinação maniqueísta de comportamentos considerados adequados à infância e ao desempenho escolar. Além disso, pesa sobre a prescrição do medicamento todas as circunstâncias que o envolvem como mercadoria e a seus fabricantes, os grandes laboratórios farmacêuticos, como corporações sujeitas a marcantes interesses econômicos, dos quais as relações com os profissionais especializados não estão isentas.

Atualmente, é comum que crianças sem queixas quanto à Saúde Mental, porém com recusas e dificuldades de adaptação a normas sociais ou a condutas da instituição escolar, sejam encaminhadas para uma avaliação com especialistas em psicologia ou em psiquiatria. Esse tem sido o âmbito da proliferação de diagnósticos de TDAH e de tratamentos estereotipados, que vão desde a prescrição única de psicoterapias individuais até a administração abusiva de medicações psicofarmacológicas operadas por simples respostas a tabelas de sintomas sindrômicos. Esses tratamentos, que por vezes se prolongam por tempo longo e indeterminado, não podem ser considerados isentos de efeitos indesejados e de 
riscos de estigmatização nem sempre menores que aqueles que se pretendia remediar.

Consultar especialistas é, por certo, uma medida prudente e adequada. Mas ninguém pode isentar-se da responsabilidade de uma cuidadosa ponderação sobre o enquadramento clínico dado ao problema e sobre as medidas terapêuticas recomendadas. É sempre conveniente considerar o perigo da comodidade enganosa de certa desresponsabilização da escola e dos pais, que já teriam delegado aos especialistas a responsabilidade de medicar e corrigir problemas infantis cujo enfrentamento delicado e responsável nem sempre é isento de dúvidas e inquietações. Dessa forma, ao invés de oferecer cuidado, essa atitude de delegação de responsabilidade pode constituir-se na permissão para que a subjetividade dessas crianças possa ser apropriada por discursos patologizantes e condutas de medicação cujos riscos devem ser considerados. 


\section{REFERÊNCIAS}

AGUIAR, A. A. Entre as ciências da vida e a medicalização da existência: uma cartografia da psiquiatria contemporânea. Revista Estados Gerais da Psicanálise, 2003. Disponível em:

<http://www.estadosgerais.org/mundial rj/download/2d Aguiar 47130903port.pdf>. Acesso em: 28 ago. 2007.

AMARANTE, P. Saúde Mental e Atenção Psicossocial. Rio de Janeiro: Editora Fiocruz, 2007.

ANGEL, M. A verdade sobre os laboratórios farmacêuticos. Rio de Janeiro: Record, 2007.

BARROS, J. A. C. et. al. Os fármacos na atualidade: antigos e novos desafios. Brasília: ANVISA, 2008.

BIRMAN, J. Mal estar na atualidade: a psicanálise e as novas formas de subjetivação. 2.ed. Rio de Janeiro: Civilização Brasileira, 2000.

BIRMAN, J. A psiquiatria como discurso da moralidade. Rio de Janeiro: Graal, 1978.

BOGOCHVOL, A. Sobre a psicofarmacologia. In: MAGALHÃES, M. C. R. (Org.). Psicofarmacologia e Psicanálise. São Paulo: Escuta, 2001.

BRZOZOWSKI, F. S.; CAPONI, S. Transtorno de Déficit de Atenção com Hiperatividade: comportamentos anormais, normalização e controle social. In: CAPONI, S. et. al. (Org.). Medicalização da vida: ética, Saúde Pública e indústria farmacêutica. Palhoça: Editora Unisul, 2010.

CAPONI, S. Da herança à localização cerebral: sobre o determinismo biológico de condutas indesejadas. Physis: Revista de Saúde Coletiva, Rio de Janeiro, v. 17, n.2, p. 342-352, 2007.

CARTILHAS DA ABDA. Cartilha para profissionais de educação: perguntas e repostas sobre TDAH, 1999. Disponível em: <http://www.tdah.org.br/br/aabda/cartilhas-da-abda.html>. Acesso em: 05 mai. 2011. 
CASTEL, R. A ordem psiquiátrica: a idade de ouro do alienismo. Rio de Janeiro: Graal, 1978.

COLLARES, C. A. L.; MOYSÉS, M. A. A. A transformação do espaço pedagógico em espaço clínico: a patologização da educação. Revista Idéias, São Paulo, n. 23, p. 25-31, 1994.

CORDEIRO, H. A indústria da saúde no Brasil. 2.ed. Rio de Janeiro: Graal, 1985.

FERRAZZA, et. al. A banalização da prescrição de psicofármacos em um Ambulatório de Saúde Mental. Revista Paidéia, Ribeirão Preto, v. 20, n. 47, p. 381-390, 2010.

FOUCAULT, M. O poder psiquiátrico: curso dado no Collège de France (19731974). São Paulo: Martins Fontes, 2006.

FOUCAULT, M. A verdade e as formas jurídicas. Rio de Janeiro: Nau Ed., 2001.

FOUCAULT, M. Microfísica do Poder. Rio de Janeiro: Graal, 1979.

GUARIDO, R.; VOLTOLINI, R. O que não tem remédio, remediado está? Educação em Revista. Belo Horizonte, v. 25, n. 1, p. 239-263, abr., 2009. Disponível em: <http://www.scielo.br/pdf/edur/v25n1/14.pdf>. Acesso em: 26 nov. 2010.

GUARIDO, R. A medicalização do sofrimento psíquico: considerações sobre o discurso psiquiátrico e seus efeitos na Educação. Educação e Pesquisa, São Paulo, v. 33, n.1, p. 151-161, jan/abr. 2007. Disponível em:

$<$ http://www.scielo.br/scielo.php?script=sciarttext\&pid=S1517>. Acesso em: 30 jun. 2008.

GOMES, M. et. al. Conhecimento sobre o transtorno do déficit de atenção/ hiperatividade no Brasil. Jornal Brasileiro de Psiquiatria, São Paulo, v. 56, n. 2, p. 94-101, set. 2007. Disponível em:

<http://www.scielo.br/pdf/jbpsiq/v56n2/a04v56n2.pdf>. Acesso em: 28 nov. 2010.

LEGNANI, V. N.; ALMEIDA, S. F. C. A construção diagnóstica de Transtorno de Déficit de Atenção/Hiperatividade: uma discussão crítica. Arquivos Brasileiros de Psicologia, Rio de Janeiro, v. 60, n. 1, p. 2-13, 2008. Disponível em: <www.psicologia.ufrj.br/abp/>. Acesso em: 22 set. 2008. 
MAIA, C.; VEREJÃO, C. Perturbação de hiperactividade com Défice da Atenção: um guia para professores. Lisboa: Associação Portuguesa da Criança Hiperactiva, 2002. $32 \mathrm{p}$.

ORTEGA, F. et. al. A ritalina no Brasil: produções, discursos e práticas. Revista Interface, v. 14, n. 34, p. 499-512, 2010.

PETRY, A. et. al. Hiperatividade: características e procedimentos básicos para amenizar as dificuldades. Revista do Professor, v. 15, n. 58, p. 47-48, 1999.

QUINET, A. (Org.). Psicanálise e Psiquiatria: controvérsias e convergências. Rio de Janeiro: Rios Ambiciosos, 2001.

ROHDE, L. et. al. Transtorno de déficit de atenção/ hiperatividade. Revista Brasileira de Psiquiatria, São Paulo, v. 22, n. 2, p. 7-11, 2000.

ROHDE, L. A.; BENCZIK. Transtorno de Déficit de Atenção e Hiperatividade: o que é? Como ajudar? Porto Alegre: Artes Médicas, 1999.

RODRIGUES, J. T. A medicação como única resposta: uma miragem do contemporâneo. Revista Psicologia em Estudo, Maringá, v.8, n.1, p.13-22, jan./jun 2003.

TELLES, J. S. S. Psicanálise em debate. Psychiatry On-line Brazil, v.5, n.3, 2000. Disponível em: <http://www.polbr.med.br/ano00/psi0300.php\#2>. Acesso em: 21 mai. 2007.

Artigo:

Recebido em: 15/02/2011

Aceito em: 21/06/2011

R. Inter. Interdisc. INTERthesis, Florianópolis, v.8, n.2, p. 237-251, Jul./Dez. 2011 